\title{
EFECTOS DE HISTAMINA SOBRE LA MOTILIDAD DEL SACO VENTRAL DEL RUMEN FETAL BOVINO
}

\author{
José Luis Arias B. (MV), Lázaro Zurich S. (MV), \\ Enrique Córdova P. (MV)

\section{EFFECTS OF HISTAMINE ON THE MOTILITY OF FETAL BOVINE RUMEN VENTRAL SAC}

\begin{abstract}
Response of in vitro histamine administration on bovine fetal rumen strips from different developmental stages were analysed. The contractile response appears in earlier fetuses 2.0 to 3.9 months) and increases until at term fetuses ( 8.0 to 8.9 months). Chlorpheniramine partially blocked the effects of histamine, while cimetidine did not. It is concluded that $H_{1}$ receptors may be involved in the complex regulations of tone and contractile response of fetal ruminal smooth muscle from 2.0 months on.
\end{abstract}

El conocimiento de la motilidad digestiva de los rumiantes ha precisado de numerosas investigaciones con el fin de establecer los mecanismos que modulan e integran los fenómenos motores, cuya importancia en los procesos digestivos del rumiante es conocida (Duncan, 1953; Comline y Message, 1965; Phillipson y Ash, 1965; Titchen y Reid, 1965; Seller y Stevens, 1966; Carr y Cols., 1970; Ruckebush, 1970; Harding y Leek, 1971; Kay y Ruckebush, 1971; Ruckebush y Kay, 1971; Asai, 1973; Church, 1974; Leek y Harding, 1975; Taneike y Ohga, 1975).

Desde hace algún tiempo se han establecido las principales sustancias que participan en los procesos inhibitorios o excitatorios de la fibra lisa digestiva del rumiante y al respecto se conoce el rol que desempeñan en el rumiante adulto los neurotransmisores autonómicos colinérgicos (Dussadier y Navarro, 1953; Brunaud y Navarro, 1954; Zurich y Cols. 1964), adrenérgicos (Dussadier y Navarro, 1953; Brunaud y Navarro, 1954; Nekrasova y Evdokinov, 1957; Paz de la Vega y Cols, 1968) y de otras sustancias como 5-hidroxitriptamina (Zurich y Cols., 1965) e histamina (Ohga y Taneike, 1978).

Con el fin de caracterizar la respuesta de diversos neurotransmisores y sustancias putativas durante el desarrollo, se han estudiado anteriormente las características de las respuestas motoras

Departamentos de Ciencias Biológicas Animales y Ciencias Clínicas.

Facultad de Ciencias Veterinarias y Pecuarias.

Universidad de Chile. Casilla 2, Correo 15.

Santiago, Chile. de la fibra muscular lisa digestiva del rumiante, en etapas de desarrollo prenatal relacionándolas con las correspondientes observaciones realizadas en el adulto.

Es así como han sido comunicados efectos de: colinérgicos (Arias y Cols., 1979a), catecolaminas (Arias y Cols., 1980c), 5-hidroxitriptamina (Arias y Cols., 1980d), prostaglandinas (Arias y Cols., 1981), sobre la motilidad del rumen fetal bovino en diferentes períodos de desarrollo.

La mayor parte de las observaciones farmacológicas coinciden con estudios ultraestructurales realizados en rumen fetal, en el sentido que parece existir algún tipo de relación entre el momento en que se establece el desarrollo papilar y la mayor susceptibilidad de la fibra lisa digestiva a la acción excitatoria de neurotransmisores y otras sustancias (Arias y Cols., 1978; Arias y Cols., 1979; Arias y Cols., 1980a, b; Arias y Cols., 1986).

Se acepta en la actualidad que los efectos de histamina, a nivel de la fibra muscular lisa, son el producto de la activación de receptores farmacológicos denominados $\mathrm{H}_{1}$, y sus efectos a nivel de la secreción gástrica corresponden a la participación de receptores denominados $\mathrm{H}_{2}$. Esta caracterización corresponde al antagonismo que ejercen bloqueadores específicos (Ash y Schild, 1966; Black y Cols., 1972; Eyre, 1973; Dousa y Code, 1974; Harris y Cols., 1975; Botting, 1975; Brimblecombe y Cols., 1975). Las acciones de histamina sobre la fibra muscular digestiva del rumen en animales adultos ha sido establecida in vitro (Duncan, 1954; Sanford, 1961; Ohga y Taneike, 1978) e in vivo (Dougherty, 1942; Clark, 1950) y 
los resultados no son concluyentes, observando algunos autores fenómenos de contracción y relajación, siendo las acciones de relajación atribuibles a la excitación $\mathrm{H}_{1}$ (Ohga y Taneike, 1978; Taneike, 1979; Ruckebusch y Soldani, 1983).

En el presente trabajo se estudia la reactividad de la pared ruminal bovina frente a histamina sola $y$ en presencia de antagonistas de receptores $\mathrm{H}_{1}$ y $\mathrm{H}_{2}$ durante diferentes etapas del desarrollo on togenético.

\section{MATERIAL Y METODOS}

Las muestras, obtenidas de plantas faenadoras de carnes, consistieron en saco ventral de rumen fetal bovino entre 2 a 9 meses de edad gestacional. La edad se obtuvo según el método de Roberts (1956). Según modelos de trabajos anteriores (Arias y Cols., 1979), los fetos se clasificaron en cuatro grupos, a saber: grupo 1 , de 2,0 a 3,9 meses; grupo 2, de 4,0 a 5,9 meses; grupo 3, de 6,0 a 7,9 meses; y grupo 4, de 8,0 a 8,9 meses de edad.

En el laboratorio, el saco ventral se dividió en tiras de $3 \mathrm{~cm}$ de largo por $0,5 \mathrm{~cm}$ de ancho y fueron colocadas en baño de órgano aislado de 30 $\mathrm{ml}$ con solución Krebs modificada* (Arias y Cols., 1979), a $390 \mathrm{C}$ y burbujeo continuo de $95 \%$ de $\mathrm{O}_{2}$ y $5 \%$ de $\mathrm{CO}_{2}$.

Los registros de motilidad de las preparaciones se obtuvieron fijando el extremo inferior al codo del asa de vidrio $y$, por su extremo superior, se conectó al transductor de tensión Universal UC-3 Statham y luego a un polígrafo inscriptor Gilson M5P. Las calibraciones se realizaron con pesas de 0,5 y $1,0 \mathrm{~g}$ con el fin de obtener deflexiones de la aguja necesaria para la conversión de $\mathrm{mm}$ de altura en $\mathrm{g}$ de tensión. Las preparaciones se mantuvieron por 60 minutos en la solución del baño aislado, para su estabilización y posteriormente se sometieron a la acción de los diferentes fármacos en estudio.

Los fármacos utilizados, en solución acuosa, fueron: clorhidrato de histamina (Sigma, Saint Louis), malea to de cloroprofenpiridamina (Laboratorio Chile, Santiago), cimetidina (droga pura, Laboratorio Chile, Santiago). Las sustancias utilizadas para la preparación de la solución Krebs modificada fueron todas de grado p.a. Merck Darmstadt.

La secuencia de agregaciones y concentraciones de drogas en el baño y otros aspectos, se detallan en el capítulo correspondiente a resultados. Las modificaciones del tono se expresan por diversos parámetros, tales como: media, error estándar y

* (g/l): $\mathrm{NaCl} 6,92 ; \mathrm{KCl} \quad 0,35 ; \mathrm{CaCl}_{2} \quad 0,50 ; \mathrm{MgSO}_{4} \quad 0,14$; $\mathrm{NaHCO}_{3} 2,10 ; \mathrm{NaH}_{2} \mathrm{PO}_{4}$ 0,32; glucosa 2,0 . coeficiente de regresión. Con el fin decomparar grupos se realizó análisis de covarianza y prueba de Scheffé (Calzada, 1954; Snedecor y Cochran, 1967; Astudillo y Cols., 1968).

\section{RESULTADOS}

\section{Efectos de histamina:}

En preparaciones con y sin movimientos espontáneos, la agregación de histamina en concentra. ciones, por duplicación $\mathrm{y}$ sin lavado intermedio desde 0,08 hasta $5,33 \mathrm{~g}$ por $\mathrm{ml}$ de solución en el baño, provocaron una respuesta contrictora en todas las preparaciones correspondientes a los diferentes periodos de desarrollo.

Analizando la magnitud de esta acción en los diferentes grupos experimentales se observó que los mayores efectos se presentaron en las etapas avanzadas del desarrollo fetal, esto es, entre 8,0 a 8,9 meses, tal como es posible apreciar en la figura 1 .

\section{Efectos de histamina en presencia de un antagonista de receptores histaminérgicos $\mathrm{H}_{1}$ : Cloroprofenpiridamina}

Establecido el efecto constrictor o de aumento del tono de histamina en la fibra lisa digestiva del rumen fetal bovino, nos pareció de interés conocer si estos efectos correspondían a un tipo de activación de receptores ligados a la contracción y clasificados como $\mathrm{H}_{1}$. Con este fin, se agregó cloroprofenpiridamina en concentración de $1 \mu \mathrm{g}$ por $\mathrm{ml}$ de baño y que se mantuvo en incubación durante 15 minutos en contacto con la preparación $\mathrm{y}$, posteriormente, se realizaron las agregaciones de histamina en igual forma y concentración descritas en el primer párrafo de resultados (vide supra). En estas condiciones cloroprofenpiridamina ejerce un efecto antagónico parcial, que fue observado en los diferentes grupos experimentales y la magnitud del antagonismo fue mayor en los grupos de desarrollo intrauterino avanzado, como se observa en la figura 1.

\section{Efectos de histamina en presencia de un antagonista de receptores $\mathbf{H}_{2}$ : Cimetidina}

Se realizaron experimentos adicionales utilizando cimetidina, droga que se ha clasificado como antagonista de los efectos de histamina en los receptores $\mathrm{H}_{2}$ y que no participan en los mecanismos que regulan la motilidad de la fibra lisa digestiva o lo hacen relajándola. De este modo, cimetidina se agregó en concentraciones de 16,6 $\mu \mathrm{g}$ por $\mathrm{ml}$ de baño y que se mantuvo por 15 minutos en contacto con la preparación, para posteriormen- 


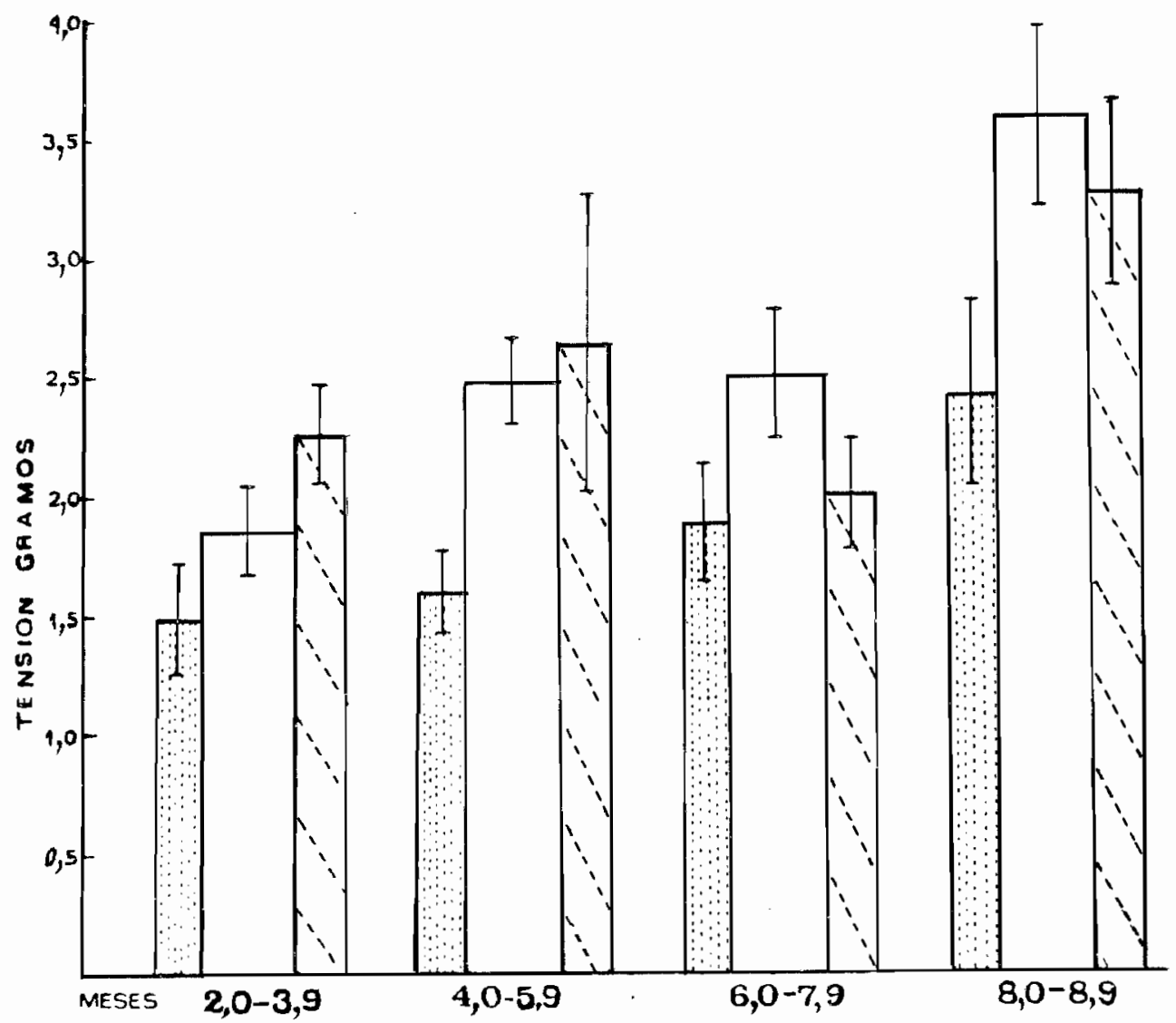

Figura 1. Desarrollo de la reactividad farmacológica del saco ventral ruminal del feto bovino a la histamina sola y en presencia de antagonistas $\mathrm{H}_{1}$ y $_{2}$.

Cada columna representa la media \pm error estándar de un promedio de 17 muestras $(n=17)$ en presencia de concentraciones submáxima de histamina $(2,66 \mu \mathrm{g} / \mathrm{ml}$ de baño

$\square=$ Histamina

Histamina en presencia de Cloroprofenpindamina $\left(\mathrm{H}_{1}\right)$.

= Histamina en presencia de Cimetidina $\left(\mathrm{H}_{2}\right)$.

te agregar la serie secuencial del agonista de igual forma que la descrita en el primer párrafo de resultados (vide supra).

En la figura 1 se puede observar que cimetidina no fue capaz de modificar los efectos constrictores de histamina, hecho que se apreció más claramente en las muestras correspondientes a desarrollo inicial (2,0 a 3,9 meses) y desarrollo medio (4,0 a 5,9 meses).

\section{DISCUSION}

A diferencia de los resultados reportados por Ohga y Taneike (1978) en rumen adulto in vivo, luego de la administración intravenosa, donde describen respuestas de contracción, relajación o respuestas bifásicas, nuestros resultados indican que durante el desarrollo, al menos en las edades estudiadas, el rumen fetal responde a la adición de histamina con contracción solamente. La magnitud de la respues- ta, al igual que en rumen adulto (Ohga y Taneike, 1978 ) es proporcional a las concentraciones de histamina utilizadas.

En general, se ha aceptado que los fenómenos de contracción en la fibra muscular lisa producidos por histamina son resultado de la activación de receptores $\mathrm{H}_{1}$ (Arunlaskshana y Schild, 1959; Ash y Schild, 1966; Botting, 1975; Hill y Cols., 1977; Ohga y Taneike, 1978). El efecto del antagonismo parcial de las contracciones producido por el antagonista $\mathrm{H}_{1}$, cloroprofenpiridamina, descrito en el presente trabajo, indicaría claramente que son justamente los receptores $\mathrm{H}_{1}$ los que estarían involucrados en las manifestaciones contráctiles de la pared ruminal fetal frente a histamina. E1 hecho que el antagonismo no sea absoluto, podría atribuirse a la concentración del antagonista utilizada.

La ausencia de respuestas de relajación sola o asociada a contracción descritas frente a histamina, podría ser debido a enmascaramiento de recepto- 
res $\mathrm{H}_{2}$ o a la ausencia de ellos durante el desarrollo. Debemos descartar aquí la interconversión de receptores $\mathrm{H}_{1}$ en $\mathrm{H}_{2}$ y viceversa dependientes de la temperatura (Kenakin y Cols., 1974), por cuanto nuestras condiciones experimentales fueron a temperaturas constante de $390 \mathrm{C}$.

En ol presente trabajo, cimetidina, antagonista de receptores $\mathrm{H}_{2}$, (Brimblecombe y Cols., 1975; Bolton, 1979), parece aumentar las respuestas contráctiles en fetos de 2,0 a 5,9 meses de edad. Si se acepta que los receptores $\mathrm{H}_{2}$ provocan una respuesta de relajación a través de liberación de catecolaminas (Everett y Mann, 1967; Tozzi, 1973; Chand y Eyre, 1975), es de esperar que su bloqueo permita una mejor expresión de los efectos de contracción mediado por $\mathrm{H}_{1}$. Este fenóneno ha sido comunicado en rumen adulto (Ohga y Taneike, 1978).

A medida que avanza el desarrollo, cimetidina no parece afectar las respuestas contráctiles de histamina. Esto podría ser explicado por una ausencia absoluta de receptores $\mathrm{H}_{2}$ o, lo que parece más probable, que los receptores $\mathrm{H}_{2}$ presentes no puedan exteriorizar su respuesta dada la liberación de catecolaminas que ocurren naturalmente durante el desarrollo (Arias y Cols., 1980a, c), lo que lleva a una depleción de ellas en periodo perinatal.

\section{RESUMEN}

En preparaciones aisladas de saco ventral del rumen fetal bovino, se estudian los efectos de histamina en las diferentes etapas del desarrollo ontogénico. La respuesta obtenida en el músculo liso ruminal es de contracción o aumento del tono; estos efectos aparecen en etapas tempranas del desarrollo fetal, 2,0 a 3,9 meses, alcanzando su efecto máximo en las últimas etapas del desarrollo, 8,0 a 8,9 meses.

Cloroprofenpiridamina, antagonista de receptores $H_{1}$, bloqueó parcialmente los efectos de histamina. Cimetidina, antagonista de receptores $\mathrm{H}_{2}$, no fue capaz de bloquear los efectos constrictores de histamina, al menos en las etapas inicial y media del desarrollo intrauterino, 2,0 a 5,9 meses, siendo inconstantes las acciones en el período más avanzados. Los hechos anteriores permiten caracterizar, de un modo preliminar, la presencia de receptores $\mathrm{H}_{1}$ en la fibra muscular lisa del rumen en períodos fetales de su desarrollo.

\section{REFERENCIAS}

ARIAS, J.L.; R. CABRERA, A. VALENCIA. Observations on the histological development of the bovine rumen papillae. Morphological changes due to age. Zentralbl. Veterinäermed. C. 7: 140-151. 1978.
ARIAS, J.L.; L. ZURICH, S. PEDREROS. Cholinergic reactivity of the bovine fetal ruminal wall in vitro. Vet. Sc. Commun. 3: 141-145, 1979a.

ARIAS, J.L.; M.S. FERNANDEZ, R. CABRERA. Distribution and nature of desmosomes in the bovine ruminal epithelium. Acta Anat. 105: 309-313, $1979 \mathrm{~b}$.

ARIAS, J.L.; M.S. FERNANDEZ, R. CABRERA. Glycogen in the epithelium of the bovine fetal rumen. Acta Anat., 106: 216-222, 1980a.

ARIAS, J.L.; E. VIAL, R. CABRERA. Observations on the histogenesis of the bovine ruminal papiliae. Am. J. Vet. Res., 41: 174-178, $1980 \mathrm{~b}$.

ARIAS, J.L.; L. ZURICH, F. COX. Efecto de catecolaminas en la pared del rumen fetal bovino. Arch. Med. Vet. 12: 252-257, 1980c.

ARIAS, J.L.; L. ZURICH, J. BASTIAS. Motor responses to 5-HT of the bovine rumen wall in vitro during fetal development. Pharmac. Res. Commun. 12: 975-985. 1980d.

ARIAS, J.L.; L. ZURICH, C.M. GONZALEZ. Effect of prostaglandin $\mathrm{F}_{2}$ alpha on the bovine ruminal wall in vitro. Vet. Res. Commun. 5: 151-158. 1981.

ARIAS, J.L.; E. GONZALEZ, E. VIVANCO, J.M. MOLINAS. Changes in conformation of collagen during development of bovine ruminal mucosa. Zentralbl. Veterinåermed. C. 30: 223-232, 1983.

ARLAS, J.L.; E. GONZALEZ, J. JIMENEZ. Conformation of interstitial collagen of bovine rumen and skin. J. Vet. Med. A. 33: 66-72, 1986.

ARUNLAKSHANA, O.; H.O. SCHILD. Some quantitative use of drug antagonists. Br. J. Pharmacol. Chemother. 14: 48-58, 1959.

ASAI, T. Development processus of reticulo-rumen motility in claves. Jap. J. Vet. Sci. 35: 239-252, 1973.

ASH, A.S.F.; H.O. SCHILD. Receptors mediating some actions of histamine. Br. J. Pharmacol. Chemother. 27: 427-439, 1966.

ASTUDILLO, V.; R. LOYOLA, M.A, MORALES, C. ORREGO, M. TORO. Elementos de bioestadística. Facultad de Ciencias Pecuarias y Medicina Veterinaria, Universidad de Chile, 1968.

BLACK, J.W.; W.A.M. DUNCAN, C.J. DURANT, C.R. GANELLIN, E.M. PARSON. Definition and antagonism of histamine $\mathrm{H}_{2}$ receptors. Nature 236: 385-390, 1972.

BOLTON, T.B. Mechanism of actions of transmitters and other substance on smooth muscle. Physiol. Rev. 59: 606-718, 1979

BOTTING, J.H. Sensivity of neonatal rabbit ileum to histamine. Br. J. Pharmacol. 53: 428-429, 1975.

BRIMB LECOMBE， R.W.; W.A.M. DUNCAN， C.J. DURANT, C.R. CANELLIN, M.E. PARSON, J.W. BLACK. The pharmacology of cimetidine a new histamine $\mathrm{H}_{2}$-receptors antagonist. Br. J. Pharmacol. 53: 435-436. 1975.

BRUNAUD, M.; J. NAVARRO. Remarques sur l'action du nerfs splanchnique et de l'adrenaline sur la motricité gastrique du mouton, Bull. Acad. Vet. (Paris) 26: 597-604, 1953.

BRUNAUD, M.; J. NAVARRO. Action sur la motricité des estomacs du mouton de quelques esters stables de la Choline. Bull. Acad. Vet. (Paris) 27: 213-217, 1954.

CALZADA, J. Métodos estadísticos para la investigación. 2da. ed. Lima, 1954

CARR, D.H.; L.M. MCLEAY, D.A. TTTCHEN. Factors affecting reflex responses of ruminant stomach. In: Phillipson, A.T., ed. Physiology of digestion and metabolism in the ruminant. England, Oriel Press, 1970. pp. 35-41. 
CLARK, R. The paralytic action of histamine on the ruminal musculature. J.S. Afr. Vet. Med. Assoc. 31: 13-17, 1950 .

COMLINE, R.S.; M.A. MESSAGE. The neuromuscular physiology of the ruminant stomach. In: Dougherty, R.W., ed. Physiology of digestion in the ruminant. Washington D.C., Butterworth, 1965, pp. 78-87.

CHAND, N.; P. EYRE. Clasification and biological distribution of histamine receptors sub-types. Agents Actions. 5: 277-295, 1975.

CHURCH, D.C. Fisiología digestiva y nutrición de los rumiantes. I. Fisiología digestiva. Zaragoza, Acribia, 1974. pp. $72-84$.

DOUGHERTY, R.W. A study of drug affecting the motility of the bovine rumen. Cornell Vet. 32: 269-280, 1942

DOUSA, T.P.; C.F. CODE. Effect of histamine and its methyl derivatives on ciclic AMP metabolism in gastric mucosa its blockade by an $\mathrm{H}_{2}$ receptors antagonist. J. Clin. Invest. 53: 334-337, 1974.

DUNCAN, D.L. The ieffets of vago tomy and splanchnotomy of gastric motility in sheep. J. Physiol. (Lond.) 119: 157-169, 1953.

DUNCAN, D.L. Responses of the gastric musculature of the sheep to some humoral agents and related substances J.Physiol. (Lond.) 125: 475-487, 1954.

DUSSARDIER, M.; J. NAVARRO. Etude in vitro des action motrices excercées par l'adrenaline et l'acetvcholine sur les estomacs des bovides. J. Physiol. (Paris), 45: 569-595, 1953.

EVERETT, S.D.; S.P. MANN. Catecholamine release by histamine from the isolated intestine of the chick. Eur. J. Pharmacol. 1: 310-320, 1967.

EYRE, P. Histamine $\mathrm{H}_{2}$ receptors in the sheep bronchus and cat trachea: the action of buramide. Br. J. Pharmacol. 48: 321-323, 1973.

HARDINGS, R.H.; B.F. LEEK. The locations and activities of medullary neurones associated with ruminant forestomach motility. J. Physiol. (Lond.) 219: $587-610,1971$

HARRIS, D.W.; J.R. SMY, J.D. REED, C.W. VENABLES The effects of burimamide and metamide on basal gastric function in the cat. Br. J. Pharmacol. 53: 293-297, 1975

HILL, S.J.; J.M. JOUNG, D.H. MARRIAN. Specific binding of $3 \mathrm{H}$-mepyramine to histamine $\mathrm{H}_{1}$ receptors in the intestinal smooth muscle. Nature (Lond.) 270: 361-363, 1977

IVY, A.C.; W.H. BACHRACH. Effect of histamine on gastric secretion. In: Eichler, O.; Farh, A., ed. Handbook of experimental pharmacology. Histamine and anti-histaminics. New York, Springer-Verlag, 1966. V. 18 p. $302-308$.

JONES, L.H. Histamina y antihistamínicos. In: Farmacología y Terapéuticas Veterinarias. México, UTEHA, 1959. 295-310.

JONES, R.G. Chemistry of histamine and analogs relationship between structures and pharmacological activity. In: Eichler O., Farah, A., ed. Handbook of experimental pharmacology. Histamine and anti-histaminics. New York, Springer-Verlag, 1966. V. 18, part. 1, p. 1-2.

KAY, R.N.B.; Y. RUCKEBUSH. Movements of the stomach compartments of young bull during sucking Br. J. Nutr. 16: 301-309, 1971.

KENAKIN, T.P.; C.A. KRUEGER, D.A. COOK. Temperature-dependent interconversion of histamine $\mathrm{H}_{1}$ and $\mathrm{H}_{2}$ receptors in guineapig ileum. Nature 252: $54-55$, 1974.
LEEK, B.F.; R.H. HARDING. Sensory nervous receptors in the ruminant stomach and the reflex control of reticulo ruminal motility. In: Mc. Donald, J.W. and Warner, A.C., ed. I: Digestion and Metabolism in the ruminant. Armsdale, N.S.W. Univ. New Engl. Publ. Unit., 1975. pp. 60-76.

NEKRASOVA, M.A.; P.D. EVDOKINOV. The effects of simpathomimetic substance on the motor function of the gastrointestinal tract in the small ruminants. $\mathrm{ZH}$., OTD. VYP, Pharm. Khimioter. Sredstva. Toksikol. 5: 255-260, 1957.

OGHA, A.; T. TANEIKE. $\mathrm{H}_{1}$ and $\mathrm{H}_{2}$ receptors in the smooth muscle of the ruminant stomach. Br. J. Pharmacol. 62: 333-337, 1978.

PAZ DE LA VEGA, Y.; L. ZURICH, S. LECANNELIER. Presencia de receptores adrenérgicos en tiras aisladas de estómagos de ovinos, estudiados con drogas estimulantes y bloqueadoras. Arch. Biol. Med. Exper. 53: 92-98, 1968

PH1LLIPSON, A.T.; R.W. ASH. Phy siological mechanism affecting the flow of digeta in ruminants. In: Dougherty, R.W. ed. Physiology of digestion in the ruminant. Washington, D.C., Butterworths, 1965, pp. 97-107.

ROBERTS, S.J. Veterinary obstetrics and genital diseases. Ithaca, N.Y. Edwards Brother, 1956. p. 17.

RUCKEBUSH, Y. The electrical activity of the digestive tract of the sheep as an indication of the mechanical events in various regions. J. Physiol. (Lond.) 210: 857-882, 1970.

RUCKEBUSH Y.; R.N.B. KAY. Etude critique de la matricité gastrique chez les bovins. Ann. Rech. Vét. 2: 99-136, 1971.

RUCKEBUSCH, Y, G. SOLDANI. $\mathrm{H}_{1}$ y $\mathrm{H}_{2}$ receptors in the ovine digestive tract. J. Vet. Pharmacol. Therap. 6: 229-232, 1983.

SANFORD, J. Some response of isolated muscle from the sheep stomach. O. J1. Exp. Phisiol. 46: 167-174, 1961.

SELLER, A.S.; C.E. STEVENS. Motor functions of the ruminant forestomach. Physiol. Rev. 46: 634-661, 1966

SNEDECOR, G.M.; W.G. COCHRAN. Statistical methods. 6a. ed. Iowa, Iowa State University Press, 1967. p. 271.

TANEIKE, T.; A. OHGA. Intrinsic innervation of isolate smooth muscle of ruminant forestomach. Jap. J. Vet. Sc. 37: 301-311, 1975 .

TANEIKE, T. 5-HT potetiates contraction mediated by the intramural cholinergic nerves in the longitudinal smooth muscle of the ruminant forestomach. J. Vet. Pharmacol. Therap. 2: 59-68. 1979.

TITCHEN, D.A.; C.S.W. REID. The reflex control of the motility of the ruminant stomach. In: Dougherty, R.W. (ed.). Physiology of the digestion in the ruminant. Washington D.C., Butterworth, 1965, pp. 68-77.

TOZZI, S. The mechanism of the action of histamine on the isolated rat uterus. J. Pharmac. Exp. Ther. 187: 511-517, 1973.

ZURICH, L.; Y. PAZ DE LA VEGA, J. BRANTES, D. LEMUS, S. LECANNELIER. Acción de fármacos sobre la motilidad de tiras aisladas de estómago de ovino. I. Fárma cos colinérgicos. Arch. Biol. Med. Exper1: $229-232,1964$

ZURICH, L.; J. BRANTES, H. BURGOS, S. LECANNELIER. Acción de fármacos sobre la motilidad de tiras aisladas de estómago ovino. II. 5-Hidroxitriptamina. Arch. Biol. Med. Exper. 2: 18-21, 1965.

Recibido en noviembre de 1987, aprobado en marzo de 1988. 